\section{VALIDATION OF THE ADJUSTED GLOBAL ANTI- PHOSPHOLIPID SYNDROME SCORE IN THE ARGENTINE POPULATION}

'Lucila Garcia*, 'Sofia Velloso, 'Victoria Martire, ${ }^{1}$ Florencia Savy, ${ }^{1}$ Fernando Arizpe, ${ }^{1}$ Nadia Garcia, ${ }^{1}$ Adriana Testi, ${ }^{1}$ Claudia Pena, ${ }^{2}$ Carolina Isnardi, ${ }^{2}$ Dafne Capelusnik, ${ }^{3}$ Victoria Collado, ${ }^{4}$ Florencia Rodriguez, ${ }^{5}$ Susana Mazza, ${ }^{5}$ Yessika Soria Curi, 'Mercedes Garcia. 'HIGA San Martin La Plata; ${ }^{2}$ Instituto de Rehabilitacion Psicofisica; ${ }^{3}$ Consultorio Privado; ${ }^{4}$ Hospital Bernardino Rivadavia; ${ }^{5}$ Hospital Padilla

\subsection{6/lupus-2019-Ism.248}

Background Assessment of risk both for pregnancy morbidity and thrombosis in the presence of antiphospholipid antibodies $(\mathrm{aPL})$ is still a challenge.

Objective to assess the performance of adjusted Global Anti-phospholipid Syndrome Score (aGAPSS) in predicting thrombosis in the setting of an external cohort study in patients with Systemic Lupus Erythematosus (SLE).

Methods Consecutive SLE patients from five rheumatology centers were included. Conventional cardiovascular risk factors were recorded as well as other underlying factors for thrombosis. Immunological tests were also recorded: ANA, anti-DNA, anti-SSA/SSB, anti-RNP, anti-Sm and aPL (Lupus Anticoagulant (LA), anti-cardiolipin (aCL) and 2 Glycoprotein I (2GPI). Medications received by patients were hydroxicloroquine, aspirin and anticoagulants. aGAPSS was calculated for each patient using a point system: 1 for arterial hypertension, 3 for dyslipidemia, 4 for LA and B2GPI and 5 for aCL. The score ranges from 0 to 17 . The discriminative ability of aGAPSS was calculated by measuring the area under the receiver operating characteristic (ROC) curve (AUC). Multivariate survival analysis was performed using the proportional hazards model to identify the association of the aGAPSS cut-off value with thrombotic events adjusted to potential confounding factors. Multivariate logistic regression analysis was performed to examine the impact

Abstract 248 Table 1 Detailed report of sensitivity and specificity

\begin{tabular}{|c|c|c|c|c|c|}
\hline \multicolumn{6}{|c|}{ Correctly } \\
\hline Cut point & Sensitivity & Specificity & Classified & LR+ & LR- \\
\hline$(\geq 0)$ & $100.00 \%$ & $0.00 \%$ & $41.67 \%$ & 1.0000 & \\
\hline$(\geq 1)$ & $77.78 \%$ & $45.24 \%$ & $58.80 \%$ & 1.4203 & 0.4912 \\
\hline$(\geq 3)$ & $65.56 \%$ & $57.94 \%$ & $61.11 \%$ & 1.5585 & 0.5945 \\
\hline$(\geq 4)$ & $62.22 \%$ & $61.11 \%$ & $61.57 \%$ & 1.6000 & 0.6182 \\
\hline$(\geq 5)$ & $46.67 \%$ & $69.84 \%$ & $60.19 \%$ & 1.5474 & 0.7636 \\
\hline$(\geq 6)$ & $35.56 \%$ & $79.37 \%$ & $61.11 \%$ & 1.7231 & 0.8120 \\
\hline$(\geq 7)$ & $31.11 \%$ & $79.37 \%$ & $59.26 \%$ & 1.5077 & 0.8680 \\
\hline$(\geq 8)$ & $30.00 \%$ & $82.54 \%$ & $60.65 \%$ & 1.7182 & 0.8481 \\
\hline$(\geq 9)$ & $27.78 \%$ & $84.92 \%$ & $61.11 \%$ & 1.8421 & 0.8505 \\
\hline$(\geq 10)$ & $14.44 \%$ & $88.89 \%$ & $57.87 \%$ & 1.3000 & 0.9625 \\
\hline$(\geq 11)$ & $10.00 \%$ & $88.89 \%$ & $56.02 \%$ & 0.9000 & 1.0125 \\
\hline$(\geq 13)$ & $10.00 \%$ & $89.68 \%$ & $56.48 \%$ & 0.9692 & 1.0035 \\
\hline$(\geq 14)$ & $2.22 \%$ & $97.62 \%$ & $57.87 \%$ & 0.9333 & 1.0016 \\
\hline$(\geq 16)$ & $2.22 \%$ & $99.21 \%$ & $58.80 \%$ & 2.8000 & 0.9856 \\
\hline$(\geq 17)$ & $1.11 \%$ & $100.00 \%$ & $58.80 \%$ & & 0.9889 \\
\hline$(>17)$ & $0.00 \%$ & $100.00 \%$ & $58.33 \%$ & & 1.0000 \\
\hline & $\mathrm{ROC}$ & & \multicolumn{2}{|c|}{ Asymptotic } & \\
\hline Obs & Area & Std. Err. & \multicolumn{2}{|c|}{ [95\% Conf. Interval] } & \\
\hline 216 & 0.6312 & 0.0374 & \multicolumn{2}{|c|}{0.55792} & 0.70443 \\
\hline
\end{tabular}

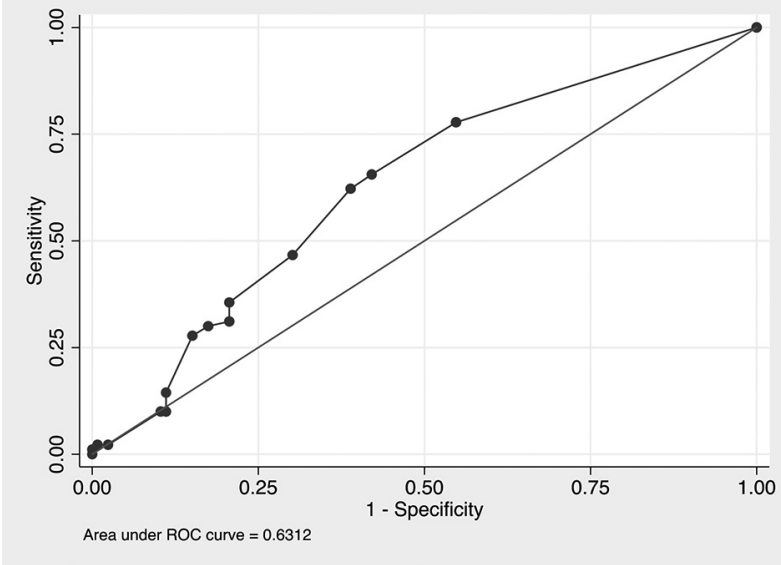

Abstract 248 Figure 1 ROC Curve

of multiple cardiovascular risk factors and laboratory parameters on the occurrence of thrombosis. A 95\% confidence interval (CI) was selected and a $\mathrm{p}$ value $<0.05$ was considered significant.

Results Information was collected from 216 SLE patients ( $89.8 \%$ women, mean age at SLE diagnosis of 31 years (SD \pm 10.5$)$. Ninety patients $(41.6 \%)$ presented thrombotic and/ or pregnancy complications. Forty-three patients (19.9\%) presented at least one thrombotic episode (53 events; 28 arterial and 25 venous thromboses). Sixty women (30.9\%) presented at least one pregnancy complication (81 events; 34 miscarriages, 28 fetal deaths and 19 premature deliveries). Median aGAPSS was significantly higher in patients who experienced a thrombotic event compared with those who had not [4 (IQR 1-9) versus 1 (IQR 0-5); p 0.001]. The AUC showed that aGAPSS 8 presented the best diagnostic accuracy $[0.63$ (CI95\% $0.55-$ $0.70) \mathrm{p} 0.03$ ] with $30 \%$ sensitivity and $82.5 \%$ specificity. Multivariate analysis indicated aGAPSS 8 was an independent predictor of thrombosis [OR: 2.1 (CI95\% 1.03-4.12) p 0.04].

Conclusions This score is a simple tool to predict risk of thrombosis in SLE patients in daily practice. The use of aGAPSS could change the non-pharmacological and pharmacological treatment in higher risk patients to improve survival. Funding Source(s): None

\section{SPLENOMEGALY AND ANTI-NUCLEAR ANTIBODIES ARE DRIVEN BY INTERFERON- STIMULATION OF B CELLS IN LUPUS-LIKE DISEASE}

${ }^{1}$ Emma J Keller*, ${ }^{2}$ Neeva Patel, ${ }^{1}$ Madeline Patt, ${ }^{3}$ Teddy Nemunaitis, ${ }^{4}$ Michael Reth, ${ }^{1}$ Ganes Sen, ${ }^{1}$ Trine Jorgensen. ${ }^{1}$ Cleveland Clinic Lerner Research Institute; ${ }^{2}$ Case Western Reserve University; ${ }^{3}$ John Carroll University; ${ }^{4}$ Max Planck Institute of Immunobiology and Epigenetics

\subsection{6/lupus-2019-Ism.249}

Background Systemic Lupus Erythematous (SLE) is an autoimmune disease of unknown etiology affecting 5 million people worldwide. It is known that $50 \%-70 \%$ of lupus patients present with an interferon-alpha (IFN-) gene signature, and it has been shown in multiple mouse models that lupus-like disease can be abolished by IFN- receptor (IFNAR) gene deficiency. Furthermore, disease can be halted by ablating the main producers of IFN-, the plasmacytoid dendritic cells. Thus, IFN- likely has a causative role in lupus-like disease. 
Multiple immune cells express IFNAR, but it is not known what the effect of IFN- stimulation is on each cell type and how that stimulation affects symptom presentation. We hypothesize that BIFNAR mice would be specifically protected from splenomegaly, B cell activation, and ANA production, due to IFN-s known ability to enhance the antibody response. Methods To examine the role of $\mathrm{B}$ cell responses to IFNstimulation in mouse lupus-like disease, we studied B cell specific IFNAR-deficiency (BIFNAR) in the B6.Nba2 spontaneous lupus-like disease model, using flow cytometry, ELISA, qRTPCR and Immunostainings. We also immunized the mice with NP-CGG or NP-Ficoll in complete Freunds adjuvant to determine the effect of IFNAR-expression by B cells during antibody responses to exogenous antigen.

Results At four months of age, BIFNAR mice displayed slightly reduced spleen sizes, although this decrease was not significant until nine months of age. Furthermore, starting at 4 months of age, BIFNAR mice displayed reduced levels of chromatin specific ANAs along with reduced populations of plasmablasts, plasma cells and activated B cells. All other measures of disease showed no difference including total IgG and IgM production, immune complex deposition and $\mathrm{C} 3$ complement fixation in the kidney glomeruli, and glomerular size. Somewhat surprising, antibody responses to T-dependent and T-independent immunizations were also not affected.

Conclusions IFN- stimulation on B cells contributed to splenomegaly, increased B cell activation and differentiation, and subsequent production of chromatin specific ANAs, but had no specific role in the response to exogenous antigen. Thus, IFN- remains a valid therapeutic target for SLE; especially in patients presenting with high ANAs.

Funding Source(s): R01AI118774

\section{PREDICTION OF FETAL LOSS IN PREGNANT PATIENTS WITH SYSTEMIC LUPUS ERYTHEMATOSUS}

${ }^{1}$ Jiayue Wu* , ${ }^{2}$ Wei-hong Zhang, ${ }^{1}$ Jinghang Ma, ${ }^{1}$ Chunde Bao, ${ }^{3}$ Jinlin Liu, ${ }^{1}$ Wen Di. ${ }^{1}$ Renji Hospital Affiliated to Shanghai Jiao Tong University School of Medicine; ${ }^{2}$ ICRH Gent University; ${ }^{3}$ School of Public Policy and Administration Xi'an Jiaotong University
Abstract 250 Table 1 Patients' characteristics during pregnancy

\begin{tabular}{|c|c|c|c|c|}
\hline Variables & $\begin{array}{l}\text { Total } \\
\mathrm{n}=338(\%)\end{array}$ & $\begin{array}{l}\text { Live birth } \\
\mathrm{n}=300(\%)\end{array}$ & $\begin{array}{l}\text { Fetal loss } \\
\mathrm{n}=38(\%)\end{array}$ & $P$ value \\
\hline \multicolumn{5}{|l|}{ Characteristics } \\
\hline Age $(y r$, mean $\pm S D)$ & $29.5 \pm 4.0$ & $29.7 \pm 3.9$ & $28.6 \pm 4.5$ & 0.131 \\
\hline $\begin{array}{l}\text { History of SLE } \\
(y r, \text { mean } \pm S D)\end{array}$ & $5.7 \pm 4.3$ & $5.8 \pm 4.4$ & $4.5 \pm 4.1$ & 0.091 \\
\hline $\begin{array}{l}\text { History of therapeutic abortion } \\
\text { (frequency, mean } \pm \mathrm{SD} \text {, range) }\end{array}$ & $\begin{array}{l}0.04 \pm 0.2 \\
(0-2)\end{array}$ & $\begin{array}{l}0.04 \pm 0.2 \\
(0-1)\end{array}$ & $\begin{array}{l}0.08 \pm 0.4 \\
(0-2)\end{array}$ & 0.657 \\
\hline $\begin{array}{l}\text { History of spontaneous } \\
\text { abortion } \\
\text { (frequency, mean } \pm \mathrm{SD} \text {, range) } \\
\text { Region }\end{array}$ & $\begin{array}{l}0.4 \pm 0.9 \\
(0-7)\end{array}$ & $\begin{array}{l}0.4 \pm 1.0 \\
(0-7)\end{array}$ & $\begin{array}{l}0.3 \pm 0.6 \\
(0-7)\end{array}$ & 0.687 \\
\hline City & $237(70.1)$ & $210(70.0)$ & $27(71.1)$ & 0.894 \\
\hline Rural & $101(29.9)$ & $90(30.0)$ & $11(28.9)$ & \\
\hline Nullipara & $291(86.1)$ & $258(86.0)$ & $33(86.8)$ & 0.888 \\
\hline Pre-pregnancy hypertension & $10(3.0)$ & $6(2.0)$ & $4(10.5)$ & $0.016^{*}$ \\
\hline Unplanned pregnancy & $45(13.3)$ & $32(10.7)$ & $13(34.2)$ & $0.000^{*}$ \\
\hline \multicolumn{5}{|l|}{ SLE clinical features } \\
\hline Renal disorder & $97(28.7)$ & $76(25.3)$ & $21(55.3)$ & $0.000^{*}$ \\
\hline Mucocutaneous & $106(31.4)$ & $94(31.3)$ & $12(31.6)$ & 0.975 \\
\hline Hematologic disorder & $66(19.5)$ & $53(17.7)$ & $13(34.2)$ & $0.015^{*}$ \\
\hline Neurologic disorder & $5(1.5)$ & $3(1.0)$ & $2(5.3)$ & 0.181 \\
\hline Arthritis & $70(20.7)$ & $63(21.0)$ & $7(18.4)$ & 0.712 \\
\hline Serositis & $17(5.0)$ & $10(3.3)$ & $7(18.4)$ & $0.001^{*}$ \\
\hline \multicolumn{5}{|l|}{ Laboratory features during pregnancy } \\
\hline $\begin{array}{l}\text { 24h-Urinary protein } \\
(\mathrm{g}, \text { mean } \pm \mathrm{SD})\end{array}$ & $1.04 \pm 2.43$ & $0.6 \pm 1.5$ & $4.3 \pm 4.9$ & $0.000^{*}$ \\
\hline Anti-dsDNA & $261(77.2)$ & $226(75.3)$ & $35(92.1)$ & $0.020^{*}$ \\
\hline Anti-Ro/SSA & $150(44.4)$ & $132(44.0)$ & $18(47.4)$ & 0.694 \\
\hline Anti-La/SSB & $47(13.9)$ & $41(13.7)$ & $6(15.8)$ & 0.722 \\
\hline Anti-Sm & $20(5.9)$ & $16(5.3)$ & $4(10.5)$ & 0.361 \\
\hline aPL & $46(13.6)$ & $38(12.7)$ & $8(21.1)$ & 0.156 \\
\hline Hypocomplementania- $C_{3}$ & $90(26.6)$ & $62(20.7)$ & $28(73.7)$ & $0.000^{*}$ \\
\hline Hypocomplementania- $\mathrm{C}_{4}$ & $60(17.8)$ & $45(15.0)$ & $15(39.5)$ & $0.000^{*}$ \\
\hline
\end{tabular}

10.1136/lupus-2019-|sm.250

\begin{tabular}{|c|c|c|c|}
\hline Variables & Risk Factors Included Individually & Risk Factors Included Simultaneously & $\begin{array}{l}\text { Risk Factors Selected Using } \\
\text { Stepwise Regression }\end{array}$ \\
\hline & $\mathrm{OR}(95 \% \mathrm{Cl})$ & $\mathrm{OR}(95 \% \mathrm{Cl})$ & $\mathrm{OR}(95 \% \mathrm{Cl})$ \\
\hline Unplanned pregnancy & $4.33(1.71-11.00){ }^{* *}$ & $3.01(0.90-10.02)$ & $2.84(1.12-7.22)^{*}$ \\
\hline Pre-pregnancy hypertension & $6.45(1.60-26.06)^{* *}$ & $5.24(0.74-37.38)$ & - \\
\hline Hypocomplementania- $C_{3}$ & $11.43(5.15-25.33)^{* *}$ & $7.09(2.60-19.36)^{* *}$ & $5.46(2.30-12.97){ }^{* *}$ \\
\hline Hypocomplementania- $\mathrm{C}_{4}$ & $3.80(1.81-7.98)^{\star \star}$ & $0.56(0.19-1.60)$ & - \\
\hline Renal disorder & $3.58(1.76-7.28){ }^{* *}$ & $0.33(0.09-1.15)$ & - \\
\hline Hematologic disorder & $2.19(1.02-4.69)^{*}$ & $0.90(0.32-2.53)$ & - \\
\hline Anti-dsDNA & $3.62(1.07-12.17){ }^{*}$ & $2.58(0.65-10.24)$ & - \\
\hline \multicolumn{4}{|l|}{ 24h-Urinary protein } \\
\hline urinary protein $<0.3 \mathrm{~g} / 24$ hour & 1 & 1 & 1 \\
\hline $0.3 \leq$ urinary protein $<1.0 \mathrm{~g} / 24$ hour & $3.34(1.05-10.68)^{*}$ & $3.23(0.90-11.65)$ & $2.10(0.63-6.95)$ \\
\hline urinary protein $>1.0 \mathrm{~g} / 24$ hour & $14.85(6.25-35.31)^{* *}$ & $16.20(3.90-67.29)^{* *}$ & $5.89(2.30-15.06) * *$ \\
\hline
\end{tabular}

NOTE: ${ }^{*} \mathrm{p}<0.05 ;{ }^{* *} \mathrm{p}<0.01$; Multivariable models adjusted for age at conception (continuous), history of SLE (continuous), history of therapeutic abortion (continuous), history of spontaneous abortion (continuous), region (city vs rural), nullipara (nullipara vs multipara) 